\title{
Silicon-on-insulator integrated source of polarization-entangled photons
}

\author{
Laurent Olislager, ${ }^{1, *}$ Jassem Safioui, ${ }^{1, *}$ Stéphane Clemmen, ${ }^{2}$ Kien Phan Huy, ${ }^{3}$ Wim Bogaerts,${ }^{4}$ Roel \\ Baets, ${ }^{4}$ Philippe Emplit, ${ }^{1}$ and Serge Massar ${ }^{5}$ \\ ${ }^{1}$ OPERA-Photonique, CP 194/5, Université libre de Bruxelles (U.L.B.), av. F.D. Roosevelt 50, B-1050 Brussels, Belgium. \\ ${ }^{2}$ School of Applied and Engineering Physics, Cornell University, Ithaca, New York 14853, USA. \\ 3 Département d'Optique P.M. Duffieux, Institut FEMTO-ST, Unité Mixte de Recherche du CNRS 6174, Université de \\ Franche-Comté, route de Gray 16, F-25030 Besançon, France. \\ ${ }^{4}$ Photonics Research Group, INTEC, Ghent University-IMEC, Sint-Pietersnieuwstraat 41, B-9000 Ghent, Belgium. \\ ${ }^{5}$ Laboratoire d'Information Quantique, CP 225, Université libre de Bruxelles (U.L.B.), av. F.D. Roosevelt 50 , B-1050 \\ Brussels, Belgium. \\ *Corresponding authors: lolislag@ulb.ac.be and jsafioui@ulb.ac.be
}

Compiled April 3, 2013

\begin{abstract}
We report the experimental generation of polarization-entangled photons at telecommunication wavelengths using spontaneous four-wave mixing in silicon-on-insulator wire waveguides. The key component is a $2 \mathrm{D}$ coupler that transforms path entanglement into polarization entanglement at the output of the device. Using quantum state tomography we find that the produced state has fidelity $88 \%$ with a pure non-maximally entangled state. The produced state violates the CHSH Bell inequality by $S=2.37 \pm 0.19$. (C) 2013 Optical Society of America OCIS codes: 130.0130, 270.0270, 270.5565.
\end{abstract}

The ability to create, manipulate and transmit the quantum state of photons has enabled applications such as quantum key distribution, as well as foundational experiments concerning for instance quantum non locality and quantum teleportation. Polarization constitutes one of the degrees of freedom most often used to code quantum information. High-quality polarization-entangled photon-pair sources have been reported based both on $\chi^{(2)}$ (see e.g. $[1-3]$ ) and $\chi^{(3)}$ (see e.g. $[4,5]$ ) nonlinear processes. To minimize cost and footprint recent work focuses on the integration of such sources. The siliconon-insulator (SOI) platform, based on reliable and lowcost CMOS technology, is a promising avenue for integrated photon-pair sources based on spontaneous fourwave mixing, both in straight wire waveguides [6] and in ring resonators [7]. Earlier works reported time-bin entanglement $[8,9]$, and polarization entanglement based either on a non-integrated polarizing beam splitter [10] or a polarization rotator sandwiched between two nonlinear silicon wire waveguides [11]. Here we present an SOI integrated source of polarization-entangled photons in which two nanophotonic waveguides produce path entanglement that is subsequently converted at the output of the chip into polarization entanglement using a $2 \mathrm{D}$ grating coupler. We characterize the source using twophoton interferences, quantum state tomography, and Bell inequality violation.

Our SOI source (fig. 1a) was fabricated by the ePIXfab at IMEC with $193 \mathrm{~nm}$ deep UV lithography. A pump beam is coupled into the structure using a 1D grating coupler followed by a taper. A 50/50 multimode coupler [12] then splits the light into two silicon wire waveguides. The waveguides have transverse dimension $500 \mathrm{~nm} \times 220 \mathrm{~nm}$ and length $15 \mathrm{~mm}$. At the operating wavelength (telecommunication C-band) the waveguides are monomode and guide only TE (horizontal) polarization. Four-wave mixing in the waveguides leads to photon-pair production, and hence to the state $a^{\prime}\left|H_{s 1}\right\rangle\left|H_{i 1}\right\rangle+b^{\prime}\left|H_{s 2}\right\rangle\left|H_{i 2}\right\rangle$, where $s, i$ refer to signal and idler frequencies, 1, 2 refer to the first and second waveguides, and we have indicated that the polarization is horizontal. The coefficients $a^{\prime}, b^{\prime}$ take into account possible deviations from a perfect 50/50 coupler, or different losses in the two waveguides. The light propagating in the waveguides is coupled into an optical fiber using inverted tapers and a 2D grating coupler [13], see inset of fig. 1a. 2D grating couplers enable polarizationinsensitive SOI structures, as they couple the two orthogonal polarizations propagating in an optical fiber to the two TE modes propagating in two distinct silicon waveguides. They can provide extinction ratio between both polarizations higher than $18 \mathrm{~dB}$ [13]. In our case the $2 \mathrm{D}$ grating coupler converts path entanglement into polarization entanglement. The state in the optical fiber is thus $|\Psi(a, b)\rangle=a\left|H_{s}\right\rangle\left|H_{i}\right\rangle+b\left|V_{s}\right\rangle\left|V_{i}\right\rangle$, where the new coefficients $a, b$ take into account possible polarizationdependent losses of the 2D grating coupler.

The experimental setup is depicted in fig. 1b. A $1 \mathrm{~mW}$ CW laser at $1539.6 \mathrm{~nm}$ is amplified to $7 \mathrm{~mW}$ with an erbium-doped fiber amplifier (EDFA) and then spectrally filtered by a band-pass filter (BPF). Injection plus extraction losses total approximately $12 \mathrm{~dB}$, and losses in the structure are close to $3 \mathrm{~dB}$. Taking into account injection loss, the power on chip is calculated to be $1.75 \mathrm{~mW}$, which is then divided in both device arms. The output containing the transmitted pump and entangled photons is collected by a cleaved standard telecommunication fiber and passes through a band-block filter (BBF) (with an isolation of more than $110 \mathrm{~dB}$ ) to reject the pump and a wavelength division multiplexer (WDM) to 
separate signal (s) and idler (i) photons into two different fiber channels which are sent to Alice (A) and Bob's (B) stations. The signal and idler ports are respectively centered at $1530 \mathrm{~nm}$ and $1550 \mathrm{~nm}$ with a bandwidth of $20 \mathrm{~nm}$. Alice and Bob locally and independently analyze their photon using a free-space analyzer consisting of a quarter-wave plate $(\lambda / 4)$, a half-wave plate $(\lambda / 2)$ and a polarizing beam splitter (PBS). Only one output of Alice's PBS -corresponding to the vertical polarization component- is available, while both outputs of Bob's PBS are available. The photons emerging from the three outputs are directed to superconducting single-photon detectors (SPD's, Scontel, efficiency 5\%, dark-count rate $10 \mathrm{~Hz}$, time resolution $50 \mathrm{ps}$ ). Total losses from pair creation to detectors are estimated at $11.6 \mathrm{~dB}$ for Alice, and $13.5 \mathrm{~dB}$ and $14.8 \mathrm{~dB}$ for Bob's two detectors. Electronic signals from the SPD's are directed to a data-acquisition system (DAS) consisting of a time-to-digital converter (Agilent Acqiris, time resolution $50 \mathrm{ps}$ ) connected to a computer. The DAS registers the relative times $t_{\mathrm{B}_{\mathrm{V}}}-t_{\mathrm{A}_{\mathrm{V}}}$ and $t_{\mathrm{B}_{\mathrm{H}}}-t_{\mathrm{A}_{\mathrm{V}}}$ between A and B's detections, and outputs a histogram of these events. When correlated photons are present, a coincidence peak emerges from these timeresolved measurements. The illustrative histograms (inset of fig. 1b) provide examples of constructive and destructive interference (note that each time bin in the histograms is $250 \mathrm{ps}$ long). Data acquisition and treatment are entirely automated.

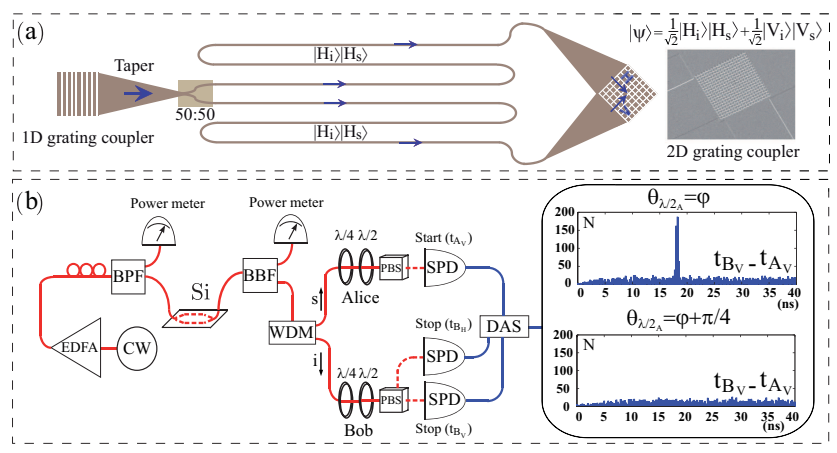

Figure 1. Panel a: schematic of the SOI chip producing polarization-entangled photon pairs. Inset: SEM image of the 2D grating coupler. Panel b: experimental setup for generating and measuring polarizationentangled photons (see text for detailed description of components). Inset: typical experimental results in the case of constructive and destructive interference.

To quantitatively analyze the results, we define the raw and net numbers of coincident events $N_{\text {raw }}=$ $\int_{t_{i}}^{t_{f}} N(t) \mathrm{d} t, N_{\text {net }}=N_{\text {raw }}-\left(t_{f}-t_{i}\right) \tau_{\text {acc }}$, where $\tau_{\text {acc }}=$ $\int_{t_{f}}^{t_{\max }} N(t) \mathrm{d} t /\left(t_{\max }-t_{f}\right)$, is the rate of accidental coincidences, and $N(t)$ is the number of coincidences at time $t=t_{\mathrm{B}_{\mathrm{V}}}-t_{\mathrm{A}_{\mathrm{V}}}$ or $t_{\mathrm{B}_{\mathrm{H}}}-t_{\mathrm{A}_{\mathrm{V}}}$. The time window for the signal has size $t_{f}-t_{i}=0.8 \mathrm{~ns}$. Outside this window, up to the maximum measurable delay $t_{\max }$, only noise is present. From these quantities we deduce that in the case of constructive interference, coincidences are measured at a rate $\approx 0.4 \mathrm{~Hz}$ and the coincidence-to-accidental ratio (CAR) is approximately equal to 8 . This rather low value is due to the $\mathrm{CW}$ operation (which increases the effect of the intrinsic noise of SOI waveguides [14]) and to the high losses from chip to detector. Dark counts are almost negligible due to the use of superconducting detectors. When all the fibers are carefully attached to guarantee stable injection and ejection and avoid polarization drift, no active power or polarization stabilization is required and measurements are repeatable for several hours.

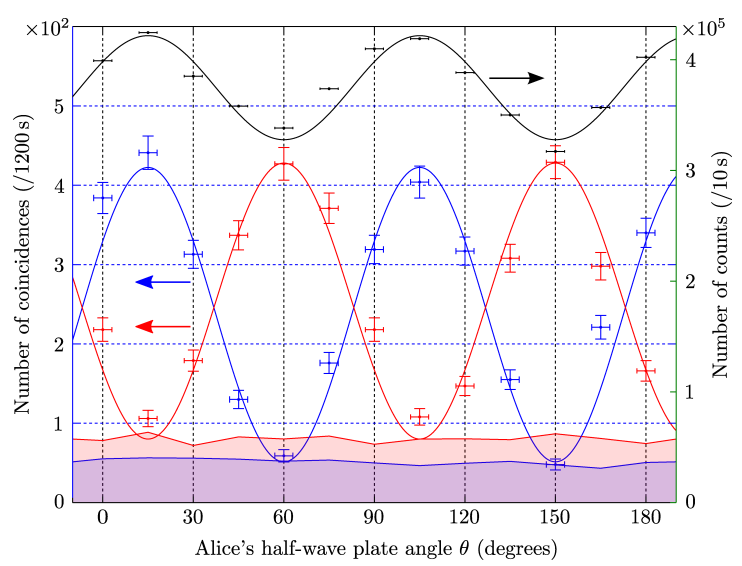

Figure 2. One and two-photon interferences. Horizontal axis is the angle $\theta$ of Alice's half-wave plate $\left(\lambda / 2_{\mathrm{A}}\right)$, on which a precision of $\pm 3^{\circ}$ is assumed. Left vertical axis is the number of coincidences between A and B's detectors registered during $20 \mathrm{~min}$. Symbols are experimental results with statistical error bars while curves are sinusoidal fits assuming a perfect net visibility. Shaded regions correspond to measured accidental coincidence rates. Blue curve corresponds to $\mathrm{A}_{\mathrm{V}} \mathrm{B}_{\mathrm{V}}$ coincidences and red curve to $\mathrm{A}_{\mathrm{V}} \mathrm{B}_{\mathrm{H}}$ coincidences. The black curve is the single-photon rate of Alice's detector as a function of $\theta$. Right vertical axis is the total number of counts registered by Alice's detector in $10 \mathrm{~s}$.

Results of two-photon interference measurements are presented in fig. 2. Coincidence rates are plotted as a function of the angle of Alice's half-wave plate. Note that because no polarization management is realized before the analyzers, all phase plate angles must be adjusted to get a good contrast. Because of noise, raw visibilities are limited to approximately $80 \%$ (vertical component) and $60 \%$ (horizontal component), while net visibilities reach respectively $99 \%$ and $90 \%$. We also measured the singlephoton rate detected by Alice (fig. 2, black curve). The fact that this curve is not perfectly flat is evidence that the produced state is not maximally entangled. This is presumably due to imperfect on-chip optical components. However, the limited visibility $(\approx 12 \%)$ shows that the produced state is not far from a maximallyentangled state. We note that non-maximally entangled states have specific applications that are not accessible 
to maximally-entangled states, see e.g. $[15,16]$.

In order to accurately characterize the produced state we realized a standard quantum state tomography analysis. We used the measurements defined in [17] and followed the maximum likelihood method described therein to evaluate the "most probable" density matrix given the statistics of coincident events measured in 16 different configurations of the analyzers. The reconstructed density matrix $\rho_{\mathrm{AB}}^{\text {(out) }}$ is then reexpressed as $\rho_{\mathrm{AB}}^{\text {(out) }}=J_{\mathrm{A}} \otimes J_{\mathrm{B}} \rho_{\mathrm{AB}}^{\text {(in) }} J_{\mathrm{A}}^{\dagger} \otimes J_{\mathrm{B}}^{\dagger}$. We optimized (numerically) the parameters of the Jones matrices $J_{\mathrm{A}}$ and $J_{\mathrm{B}}$ as well as the real numbers $a, b$ (with $a^{2}+b^{2}=1$ ) in order to maximize the fidelity $F\left(\rho_{\mathrm{AB}}^{\text {(in })}, \rho_{\mathrm{AB}}^{\text {(target) }}\right)=$

$$
\begin{gathered}
\left(\operatorname{tr}\left[\left(\sqrt{\rho_{\mathrm{AB}}^{\text {(in })}} \rho_{\mathrm{AB}}^{\text {(target) }} \sqrt{\rho_{\mathrm{AB}}^{(\mathrm{in})}}\right)^{1 / 2}\right]\right)^{2} \text {, where } \\
\rho_{\mathrm{AB}}^{\text {(target) }}=|\Psi(a, b)\rangle\langle\Psi(a, b)|=\left(\begin{array}{cccc}
a^{2} & 0 & 0 & a b \\
0 & 0 & 0 & 0 \\
0 & 0 & 0 & 0 \\
b a & 0 & 0 & b^{2}
\end{array}\right)
\end{gathered}
$$

is the density matrix of a pure non-maximally entangled state. Thus $\rho_{\mathrm{AB}}^{(\mathrm{in})}$ is our reconstruction of the state at the output of the SOI chip and $J_{\mathrm{A}}, J_{\mathrm{B}}$ our reconstruction of the polarization rotation undergone by $\mathrm{A}$ and $\mathrm{B}$ photons between the chip and the analyzers. The results of this analysis are presented in fig. 3. The reconstructed density matrix $\rho_{\mathrm{AB}}^{(\mathrm{in})}$ has a fidelity of $88 \%$ (which drops to $71 \%$ when noise is not subtracted) with the target state with $a^{2} \approx 0.6$ and $b^{2} \approx 0.4$. The fidelity to a maximallyentangled state is $87 \%$.
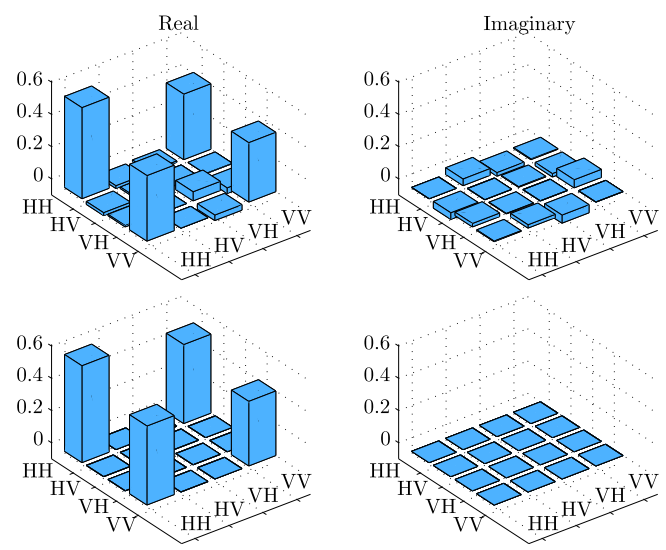

Figure 3. Upper panel: Estimated density matrix $\rho_{\mathrm{AB}}^{\text {(in) }}$ of the state produced at the output of the silicon chip. This state has $88 \%$ fidelity with the non-maximally entangled state $\sqrt{0.6}|H\rangle_{\mathrm{A}}|H\rangle_{\mathrm{B}}+\sqrt{0.4}|V\rangle_{\mathrm{A}}|V\rangle_{\mathrm{B}}$ whose density matrix is represented on the lower panel. For each density matrix, $\operatorname{Re}(\rho)$ and $\operatorname{Im}(\rho)$ are plotted on left and right respectively.

Finally, we measured the CHSH inequality [18]

$$
S=E\left(A_{1} B_{1}\right)+E\left(A_{1} B_{2}\right)+E\left(A_{2} B_{1}\right)-E\left(A_{2} B_{2}\right) \leq 2
$$

with $E=\frac{\left(N_{00}+N_{11}\right)-\left(N_{01}+N_{10}\right)}{\left(N_{00}+N_{11}\right)+\left(N_{01}+N_{10}\right)}, N_{i j}$ being the number of coincidences registered at Alice's output $i=0,1$ and Bob's output $j=0,1$. The 3 available outputs give directly the values of $N_{10}$ and $N_{11}$. To estimate $N_{00}$ and $N_{01}$, we proceed similarly to [19], using expression $E=\frac{\left(N_{0}^{\mathrm{B}}-2 N_{10}\right)-\left(N_{1}^{\mathrm{B}}-2 N_{11}\right)}{N_{0}^{\mathrm{B}}+N_{1}^{\mathrm{B}}}$, where $N_{i}^{\mathrm{B}}=N_{0 i}+N_{1 i}$, $i=0,1$, are estimated from two-photon interference measurements, see fig. 2. After carefully selecting the values of analyzer parameters for which the value of $S$ will be maximal, we measure (after subtraction of noise) $S=2.37 \pm 0.19$, thereby violating the CHSH inequality by almost 2 standard deviations.

In summary we have presented an SOI integrated source of polarization-entangled photons based on a $2 \mathrm{D}$ grating coupler. In future work the degree of entanglement of the source could be tuned on-chip by modifying the ratio of the integrated coupler. Our work confirms the relevance of SOI for integrated quantum optics.

This research was supported by the Interuniversity Attraction Poles program of the Belgian Science Policy Office, under grant IAP P7-35 "photonics@be". We thank Nam Nguyen for experimental support.

\section{References}

1. P. G. Kwiat, K. Mattle, H. Weinfurter \& A. Zeilinger, New High-Intensity Source of Polarization-Entangled Photon Pairs, Physical Review Letters 75, 4337 (1995).

2. G. Fujii, N. Namekata, M. Motoya, S. Kurimura \& S. Inoue, Bright narrowband source of photon pairs at optical telecommunication wavelengths using a type-II periodically poled lithium niobate waveguide, Optics Express 15, 12769 (2007).

3. A. Martin, A. Issautier, H. Herrmann, W. Sohler, D. B. Ostrowsky, O. Alibart \& S. Tanzilli, A polarization entangled photon-pair source based on a type-II PPLN waveguide emitting at a telecom wavelength, New Journal of Physics 12, 103005 (2010).

4. H. Takesue \& K. Inoue, Generation of polarizationentangled photon pairs and violation of Bell's inequality using spontaneous four-wave mixing in a fiber loop, Physical Review A 70, 031802(R) (2004).

5. X. Li, P. L. Voss, J. E. Sharping \& P. Kumar, OpticalFiber Source of Polarization-Entangled Photons in the 1550 nm Telecom Band, Physical Review Letters 94, 053601 (2005).

6. J. E. Sharping, K. F. Lee, M. A. Foster, A. C. Turner, B. S. Schmidt, M. Lipson, A. L. Gaeta \& P. Kumar, Generation of correlated photons in nanoscale silicon waveguides, Optics Express 14, 12388 (2006).

7. S. Clemmen, K. Phan Huy, W. Bogaerts, R. Baets, P. Emplit \& S. Massar, Continuous wave photon pair generation in silicon-on-insulator waveguides and ring resonators, Optics Express 17, 16558 (2009).

8. H. Takesue, Y. Tokura, H. Fukuda, T. Tsuchizawa, T. Watanabe, K. Yamada \& S. Itabashi, Entanglement generation using silicon wire waveguide, Applied Physics Letters 91, 201108 (2007).

9. K.-I. Harada, H. Takesue, H. Fukuda, T. Tsuchizawa, T. Watanabe, K. Yamada, Y. Tokura \& S.-I. Itabashi, 
Generation of high-purity entangled photon pairs using silicon wire waveguide, Optics Express 16, 20368 (2008).

10. H. Takesue, H. Fukuda, T. Tsuchizawa, T. Watanabe, K. Yamada, Y. Tokura \& S.-I. Itabashi, Generation of polarization entangled photon pairs using silicon wire waveguide, Optics Express 16, 5721 (2008).

11. N. Matsuda, H. Le Jeannic, H. Fukuda, T. Tsuchizawa, W. J. Munro, K. Shimizu, K. Yamada, Y. Tokura \& H. Takesue, A monolithically integrated polarization entangled photon pair source on a silicon chip, Scientific Reports 2, 00817 (2012).

12. W. Bogaerts, S. K. Selvaraja, P. Dumon, J. Brouckaert, K. De Vos, D. Van Thourhout \& R. Baets, Silicon-oninsulator spectral filters fabricated with CMOS technology, IEEE Journal of Selected Topics in Quantum Electronics 16, 33 (2010).

13. D. Taillaert, H. Chong, P. I. Borel, L. H. Frandsen, R. M. De La Rue \& R. Baets, A compact Two-Dimensional grating coupler used as a polarization splitter, IEEE Photonics Technology Letters 15, 1249 (2003).

14. S. Clemmen, A. Perret, J. Safioui, W. Bogaerts, R. Baets, S.-P. Gorza, P. Emplit \& S. Massar, Low-power inelastic light scattering at small detunings in silicon wire waveguides at telecom wavelengths, Journal of Optical Society of America B 29, 1977 (2012).

15. L. Hardy, Nonlocality for two particles without inequalities for almost all entangled states, Physical Review Letters 71, 1665 (1993).

16. M. Giustina, A. Mech, S. Ramelow, B. Wittmann, J. Kofler, J. Beyer, A. Lita, B. Calkins, T. Gerrits, S. W. Nam, R. Ursin \& A. Zeilinger, Bell violation with entangled photons, free of the fair-sampling assumption, http://arxiv.org/abs/1212.0533 (2012).

17. D. F. V. James, P. G. Kwiat, W. J. Munro \& A. G. White, Measurement of qubits, Physical Review A 64, 052312 (2001).

18. J. F. Clauser, M. A. Horne, A. Shimony \& R. A. Holt, Proposed Experiment to Test Local Hidden-Variable Theories, Physical Review Letters 23, 880 (1969).

19. J. F. Clauser \& M. A. Horne, Experimental consequences of objective local theories, Physical Review D 10, 526 (1974). 